\title{
El Hospital Varsovia de Toulouse, un proyecto del exilio español
}

\author{
The Warsaw's Hospital of Toulouse, a project of the Spanish exile
}

O Hospital Varsóvia de Toulouse, um projeto do exílio espanhol

\author{
Pablo Molanes Pérez \\ Enfermero diplomado, Máster en Innovación e Investigación en Ciencias de la Salud por la Universidad de Cádiz y estudiante \\ de $4^{\circ}$ curso de la licenciatura de Historia. \\ Cómo citar este artículo en edición digital: Molanes Pérez, P. (2013) El Hospital Varsovia de Toulouse, un proyecto del exilio \\ español. 17, 35. Disponible en: http://dx.doi.org/10.7184/cuid.2013.35.06 \\ Correspondencia: C/Benito Pérez Galdós n84 Po1-A-5. CP. 11002 Cádiz. Correo electrónico: pablo.molanesperez@alum.uca.es \\ Recibido: 24/11/2011. Aceptado: 25/12/2012
}

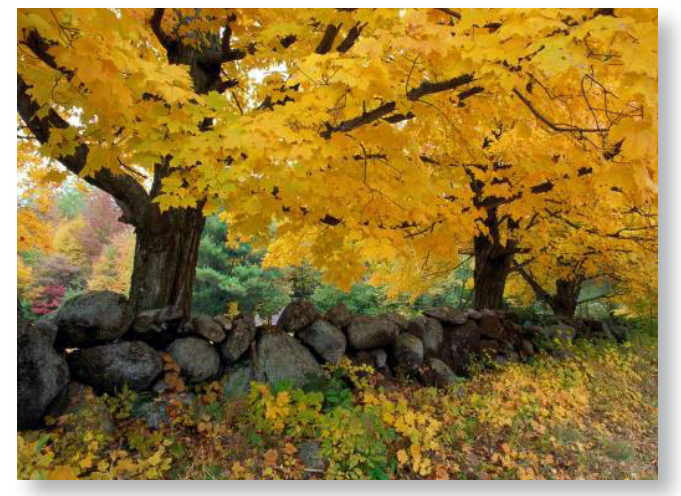

“Guerrillero español: en ti saludo a tus bravos compatriotas, por vuestro valor, por la sangre vertida por la libertad y por Francia. Por tus sufrimientos, eres un héroe francés $y$ español."

Charles de Gaulle, marzo de 1945.

\section{ABSTRACT}

The failed invasion of Arán Valley during Operation Reconquest developed in October 1944 made it necessary to establish a hospital to take care of the sick and injured from the operation. In October 1944, the Warsaw Hospital is opened in the Saint Cyprien neighborhood of Toulouse. The objective of this article is to do a critical revision of the history of the hospital during the Spanish stage, basing our reflexion on the available publications and primary sources. The presence of numerous refugees in need of medical care and the failure of the military operations contribute to the hospital's transformation into a center of free care for the exiled civil population and also a meeting place. The center receives international aid, principally from the Join Anti-Fascist Refugee Committee distributed through the humanitarian organization Unitarian Service Committee. The hospital is instituted with the aim of being a model republican hospital so as to be implemented with the return of a democratic regime in Spain. As a result of the anticommunist campaign at the onset of the cold war the hospital is accused of being a "refuge of soviet agents". On 7 September of 1950, during Operation Bolero-Paprika, 50 people are imprisoned in Toulouse, amoung them the medical staff at the Warsaw hospital. The project was cut short by the change in international politics but managed to be saved thanks to the involvement of French doctors and the economic support of the PCF, guaranteeing its survival as a welfare center until nowadays.

Keywords: Operation Bolero-Paprika, communist party, guerrilleros, Warsaw hospital, exile. 


\section{RESUMO}

O fracasso da invasão do vale de Aran, na Operação Reconquista, desenvolvida em outubro de 1944, fez necessário estabelecer um centro hospitalar para atender aos feridos e aos doentes da operação. Em outubro de 1944 inaugurou-se o Hospital Varsóvia, no bairro de Saint Cyprien, em Toulouse. O objetivo deste artigo é fazer uma revisão crítica da história do centro hospitalar durante a etapa espanhola, apoiando-se nas publicações e fontes primárias disponíveis. A presença duma grande quantidade de refugiados que necessitam de cuidados sanitários e o fracasso das operações militares contribuíram à transformação num centro de cuidados gratuitos para a povoação civil exilada e num lugar de encontro. O centro recebeu apoios internacionais, o principal a través do Join Anti-Fascist Refugee Committee, distribuídos a través da organização humanitária Unitarian Service Committee. O centro constituiu-se como um modelo de hospital republicano, com vistas à implantação do mesmo ao retornar a regime democrático à Espanha. Fruto da campanha anticomunista pelo começo da guerra fria, o centro hospitalar foi acusado de ser "refúgio de agentes soviéticos". O 7 de setembro de 1950, dentro da Operação Bolero-Paprika, foram presos em Toulouse 50 pessoas, entre elas toda a equipe médica do Hospital Varsóvia. O projeto viu-se decepado pela mudança da política internacional, mas conseguiu manter-se graças à implicação de médicos franceses e ao apoio do PCF, que garantiu a sobrevivência assistencial até hoje.

Palavras chave: Operação Bolero-Paprika, Partido Comunista, guerrilheiros, Hospital Varsóvia, exílio.

\section{RESUMEN}

El fracaso de la invasión del valle de Arán en la Operación Reconquista, desarrollada en octubre de 1944 hizo necesario establecer un centro hospitalario para atender a los heridos y enfermos de la operación. En octubre de 1944 se dotó el Hospital Varsovia, en el barrio de Saint Cyprien, en Toulouse. El objetivo de este artículo es hacer una revisión crítica de la historia del centro hospitalario durante la etapa española, apoyándose en las publicaciones y fuentes primarias disponibles. La presencia de una gran cantidad de refugiados necesitados de cuidados sanitarios y el fracaso de las operaciones militares contribuyeron a la transformación en un centro de cuidados gratuitos para la población civil exiliada y en un lugar de encuentro. El centro recibió apoyos internacionales, el principal a través de Join AntiFascist Refugee Committee, distribuidos a través de la organización humanitaria Unitarian Service Committee. El hospital se constituyó como un modelo de hospital republicano, con vistas a la implantación del mismo al retornar el régimen democrático a España. Fruto de la campaña anticomunista por el inicio de la guerra fría el centro hospitalario fue acusado de ser "refugio de agentes soviéticos". El 7 de septiembre de 1950, dentro de la Operación Bolero-Paprika fueron encarcelados en Toulouse 50 personas, entre ellas todo el equipo médico del hospital Varsovia. El proyecto se vio truncado por el viraje de la política internacional pero consiguió salvaguardarse gracias a la implicación de médicos franceses y al apoyo económico del PCF, que aseguró su continuidad como centro asistencial hasta nuestros días.

Palabras clave: Operación Bolero-Paprika, partido comunista, guerrilleros, hospital Varsovia, exilio. 


\section{INTRODUCCIÓN}

El éxodo español, consecuencia de la Guerra Civil (1936-1939) superó ampliamente a todo el conjunto de emigraciones políticas españolas de los últimos 150 años. Tras la caída de Barcelona, el 26 de enero de 1939 comenzó la larga peregrinación de exiliados hacia la frontera gala. El gobierno francés de Edouard Daladier, colapsado por la avalancha del éxodo, decidió abrir la frontera el 29 de enero y encerró a los exiliados en campos de concentración improvisados, distribuidos por todo el sur del país. Se hace difícil cifrar el número de personas exiliadas por el continuo flujo del mismo, pero el cómputo oficial recogido por las autoridades francesas de los españoles exiliados habla de 527.843 personas, de las cuales se censaron el verano de 1939 alrededor de 1500 profesionales sanitarios, entre los que se incluyen 553 médicos, 135 dentistas, 268 farmacéuticos, 503 practicantes y enfermeras y 41 optometristas. En 1945 tan sólo quedaban registrados en Francia 172 médicos, 13 dentistas, 128 practicantes y 16 farmacéuticos españoles y fueron pocos a los que se les permitieron continuar trabajando en su especialidad (1).

Las condiciones de vida en los campos de concentración eran pésimas. Según los informes sanitarios franceses casi la totalidad de los exiliados tenían parásitos, el 30 por ciento sarna, la disentería era bastante habitual y el tifus hacía estragos. Se habilitaron hospitales de campaña, así como barcos hospitales (Port Vendres), pero las autoridades sanitarias francesas se vieron desbordadas. Médicos y sanitarios exiliados participaron en el cuidado de los refugiados en los campos de concentración, bajo la supervisión de las autoridades francesas y con la carestía de medios correspondientes, ya que en muchos casos tan sólo disponían del material sanitario militar transportado por los republicanos en la retirada.

El Gobierno Republicano en el exilio organizó desde París una red de servicios médicos atendidos por sanitarios españoles por todo el país. La Cruz Roja Republicana Española estableció 90 dispensarios, distribuidos por todo el territorio francés. Tan sólo el dispensario instalado en París atendió entre 1947 y 1948 a 107.470 consultas.

Ante la situación de colapso sanitario el gobierno francés, gracias al ministro de sanidad, de filiación comunista, emitió un decreto denominado la ley Billoux del 5 de agosto de 1945, que permitía, amparándose en la convención internacional de 1933 que los profesionales sanitarios asistieran a sus compatriotas.

Muchos exiliados españoles en Francia se unieron al movimiento de resistencia interior de la Francia Libre, contribuyendo a la liberación de Francia. Según las estimaciones, fueron alrededor de 50.000 los españoles que lucharon contra los nazis en Francia. A partir del verano de 1941 los soldados exiliados constituyen la Agrupación de Guerrilleros Españoles (AGE), integrada dentro de las Fuerzas Francesas del Interior (FFI). La actuación más importante de esta organización sería la liberación de Foix, así como la liberación de Toulouse, bajo el mando de Luis Bermejo y la colaboración en la liberación de Limoges.

La Unión Nacional Española (UNE), formada por Socialistas, Republicanos y Monárquicos en el exilio lanza el 21 de agosto de 1943 una llamada a todos los españoles para terminar con el régimen de Franco, convirtiéndose en la única voz legítima en el exilio, a semejanza del Comité Français de Libération Nationale. Comienza a tomar cuerpo la necesidad de iniciar una operación contra el régimen de Franco, confiando en que la ofensiva desmora- 
lizaría al ejército franquista y provocaría una insurrección interna, para ello se organizó en mayo de 1944 la Agrupación de Guerrilleros Españoles (AGE) y se convierte a Toulouse en el centro de operaciones de la resistencia española. En lo que se ha denominado la "Operación Reconquista" la Agrupación de Guerrilleros Españoles (AGE) movilizó un conjunto que oscila entre los 5000 y los 8000 maquis, que entraron el 17 de octubre por la frontera con Navarra, Huesca y Lérida, mientras que el bloque más numeroso entró por el valle de Arán. La lucha duró 11 días, superados en número, armamento y preparación por las tropas franquistas. Será Santiago Carrillo, dirigente del PCE quien dé la orden de retirada el 29 de octubre. La destitución de Jesús Monzón de la dirección del PCE como consecuencia del desastre de la Operación Reconquista y la toma de la decisión de retirada por parte de Santiago Carrillo supondrá el ascenso de este último en la jerarquía del partido. Como consecuencia de la Operación Reconquista, a mediados de octubre de 1944 se fundó en Toulouse el Hospital Varsovia, con la finalidad de atender a los heridos de las guerrillas.

Se ha tratado en diversas publicaciones la gestión española del centro, sin embargo la lucha anticomunista primero, la opacidad de las acciones orquestadas por el partido comunista, la negligencia en la conservación documental y la perdida de los testimonios de los protagonistas al no haber sido convenientemente recogidos antes de su muerte ha dejado como resultado una historia inconclusa, sesgada y parcial.

El objetivo de este artículo es hacer una revisión sistemática de toda la bibliografía producida referente al centro hospitalario durante su dirección por sanitarios españoles, para ser reinterpretada y complementada con las fuentes primarias disponibles en los Archivos del
Hospital, municipales y departamentales, así como del Archivo de la Prefectura de la Policía de París.

El Marco Teórico Referencial de este trabajo es el de la Teoría Critica, que nace como necesidad de poner en cuestión la historia oficial, positivista, impuesta por las clases dominantes. Rescata el papel de los marginados, de los derrotados, en este caso de los exiliados, de esos sanitarios españoles expulsados primero de su país y posteriormente de su obra hospitalaria, fruto de la caza de brujas que fue la lucha anticomunista a mitad del siglo XX. Se trata de una historia no falta de rigor, pero que carece de la legitimidad que da la historia positivista, que reinterpreta como se ha escrito la historia a partir de las trazas dejadas por testimonios y documentos, teniendo en cuenta el contexto en el que se generaron y las fuerzas en conflicto que influyeron en la producción y conservación de la documentación histórica, realizando un análisis filosófico de los acontecimientos históricos. Este marco teórico requiere de la creatividad y la capacidad observadora de los historiadores para reconstruir historias posibles, paralelas u ocultadas por la historia oficial.

Si revisamos las publicaciones, la primera referencia la encontramos en el texto de David Pike Jours de gloire, jours de honte: le parti communiste d'Espagne en France depuis son arrivée en 1939 jusqu'à son départ en 1950, de 1984. Este texto dedica un capítulo completo al hospital Varsovia, haciendo por primera vez una revisión de los documentos policiales y contrastándolo con testimonios de testigos presenciales, sin embargo ofrece una visión partidista, corroborando el punto de vista policial, pese a encontrarse en la mayoría de los casos fundamentados en fuentes poco fidedignas o inciertas, tachando al centro y al personal como quinta columna del comunismo internacional. 
El siguiente trabajo de importancia en tratar el tema es el proyecto de Tesis Doctoral de Janine Garipuy, de 1987, titulado L'Hôpital Joseph Ducuing et son projet: Histoire, realite et image d'aujourd'hui que retoma la gestion española aportando testimonios directos. Diez años más tarde Dolores Villar-Basanta escribirá su memoria de D.E.A. titulada L'Hôpital Varsovie, 1944-1950, un estado de la cuestion que no logra profundizar mucho mas en el tema. Tras éste habrá que esperar otra década hasta el inicio de las investigaciones del profesor Alvar Martínez Vidal, las cuales realizaba de forma simultánea a mi primera visita a Toulouse. De sus investigaciones ha resultado la publicación en catalán del libro Exili, medicina i filantropía en el año 2010, traducido al francés el año 2011, titulado L'Hopital Varsovie. Exil, médecine et résistance (1944-1950), adoleciendo de una edición en castellano ya que se trata de un trabajo fundamental para ahondar en la historia de la etapa española del centro, reuniendo documentación sobre el hospital dispersa por distintos centros, pero como el mismo declara, se trata de un trabajo inconcluso, mas bien planteando distintos campos de trabajo que quedan por explorar.

\section{DESARROLLO DEL TEMA}

\section{Evolución del centro y apoyos internacionales.}

El hospital es fundado en octubre de 1944, con el objeto de atender a los guerrilleros heridos. Pese a la falta de documentación sobre la fundación del hospital en el artículo de Alejandro Sánchez, se menciona una carta de Celedonio Villanueva en la cual el Teniente médico Dr. Momeñe (militante del PCE) advertía al estado mayor guerrillero de la existencia de un castillo abandonado por los alemanes en el número 15 de la calle Varsovia que podía servir como instalación médica a los guerrilleros

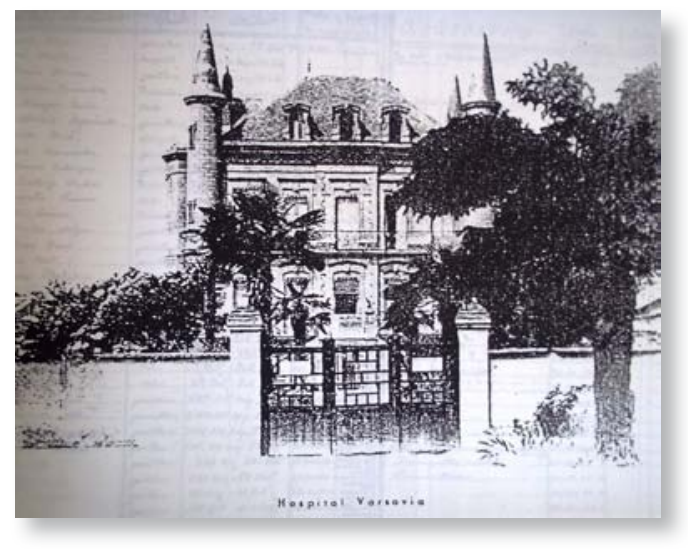

(Foto 1: Hospital Varsovia de Toulouse. Fuente: Tesis doctoral Janine Garipuy).

El Comisario del Gobierno Provisional de la República Francesa, Pierre Bertaux permitió a los guerrilleros la ocupación del castillo gracias a la mediación del secretario del PCF Henri Dupont. El hospital tomó el nombre de la calle donde se situaba, sin tener ninguna relación con la capital polaca. Según los investigadores sería una deformación de la expresión en occitano de "verse vin". Los servicios de sanidad militar francesa contribuyeron a la dotación del hospital con el material sanitario requisado a los alemanes en su repliegue. El primer hospitalizado entraría el 20 de octubre de 1944. En los tres primeros meses se atendería cerca de 200 enfermos, la mayoría provenían de las divisiones 99, 204 y 186. Contó con una capacidad inicial de 53 camas distribuidas en cinco salas y con una plantilla de 25 médicos y enfermeras exiliados, la mayoría de filiación comunista (Guerra, 2003: 204-206).

"Al hospital Varsovia van a ser evacuados la mayoría de los guerrilleros heridos durante la operación del valle de Arán. No en vano, el jefe del Servicio Sanitario de la invasión, el doctor Diego Díaz Sánchez, había sido uno de los fundadores."(2) 
El primer director del centro fue el teniente Médico José Miguel Momeñe González, en algunos trabajos aparece como Médico, pero Francisco Guerra (Guerra, 2003) lo presenta como practicante, graduado por la universidad de Valladolid en 1925.

La presencia de una gran cantidad de refugiados necesitados de cuidados sanitarios y el fracaso de las operaciones militares contribuyen a la transformación en un centro de cuidados gratuitos para la población civil exiliada y en un lugar de encuentro. Para poder funcionar como centro civil se constituye el 30 de mayo de 1945 la Amicale des Anciens FFI et Résistants Espagnols, que se encargará de la administración del centro, sirviéndose del decreto ley del 12 de abril de 1939 que regulaba las asociaciones extranjeras.

El cambio de estatus hizo que se destituyera al director militar, el Dr. Momeñe, y se nombrara director del centro al Dr. Torrubia, miembro del PSUC. El 18 de junio de 1945 ingresa, según el registro de hospitalización la primera mujer. El Essai biographique de l'hôpital Varsovie editado por el propio hospital en 1950 dice textualmente:

"Ningún enfermo tiene la impresión de estar en un hospital donde todo lo que le rodea le sea extraño: al contrario, tiene la sensación de estar cuidado en su casa y en familia; Médicos, enfermeras y personal administrativo, todos españoles, con la única preocupación del paciente que es al mismo tiempo su amigo del exilio y su compañero de lucha por la reconquista de la patria perdida." (3)

Este sentimiento de transformación del centro hospitalario en un refugio de la patria perdida queda bien reflejado en las cartas remitidas por los enfermos a la dirección del centro:

"Nunca habría pensado - nos escribe S.L. de
Septfonds- que el Hospital Varsovia fuera un auténtico rincón de nuestra patria donde encontramos no solo los cuidados necesarios, sino también el cariño de todo el personal."(4)

Desde su creación el mayor reto del hospital fue el de recaudar fondos para mantener la actividad asistencial. El Comité de Ayuda a los Republicanos Españoles, cuyo presidente fue Pablo Picasso hizo un llamamiento de ayuda al "hospital de los españoles en Francia"(5). En una biografía sobre Pablo Picasso, escrita por su nieto Olivier Widmaier afirma:

"más adelante, mucho más adelante, Pablo regaló algunos cuadros a instituciones o personas que habian ayudado a los republicanos: en 1940, al Hospital Ducuing de Toulouse, que había acogido a numerosos refugiados."(6)

Aunque la fecha que da Widmaier es errónea, ya que el Hospital no comienza a funcionar hasta 1944 es bastante plausible que Picasso donara un cuadro al hospital, ya que era una práctica muy extendida por el pintor para apoyar a las instituciones con las que simpatizaba. Tras consultar con el otro investigador, el profesor Alvar y con la actual dirección del centro hospitalario, ambos manifestaron ignorar el hecho de la donación de una pintura al hospital. Hemos intentado identificar qué cuadro pudo ser sin éxito, el vacío informativo con respecto a la donación de la pintura y su destino ulterior no dejan de ser reveladores y creemos que bien pudo ser vendido para costear los gastos del centro hospitalario o bien pudo ser requisado por el PCE o el PCF antes del cambio de dirección del hospital. En esta época pinta Picasso varios cuadros solidarizándose con la causa republicana, como "Aux espagnols morts pour la France" pintado entre 1946-1947, que en la actualidad se expone en el Museo Reina Sofía de Madrid 


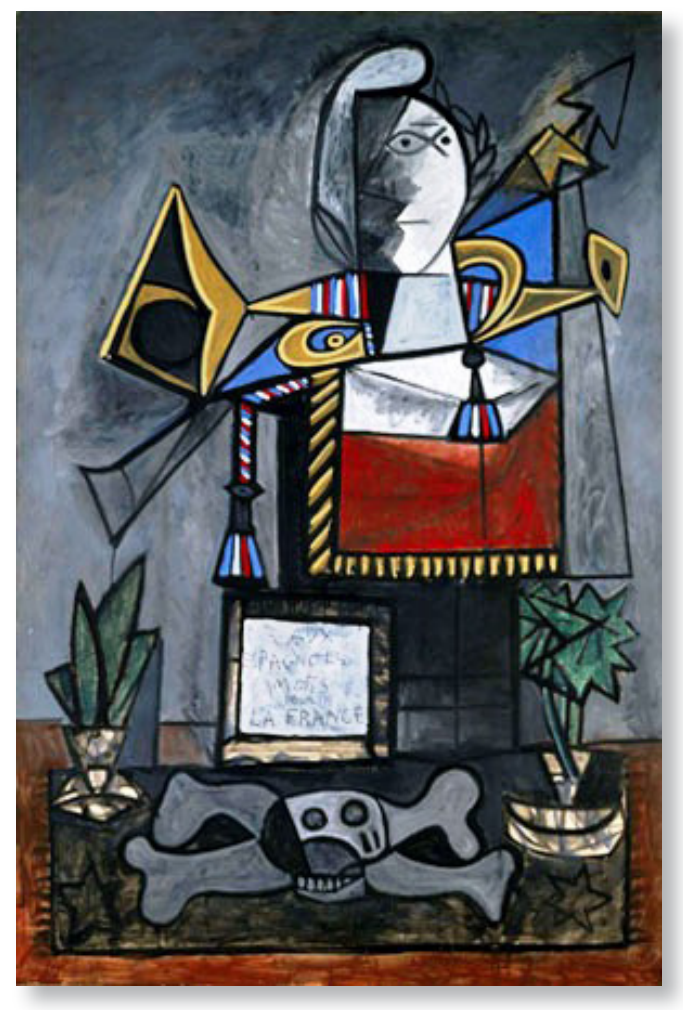

(Foto 2: Aux espagnols morts pour la France. Pablo Picasso. Fuente: Catálogo del Museo Reina Sofía).

Muchos otros contribuyeron a la causa del hospital, la Seguridad Social francesa participaba del sostenimiento del hospital, gracias a un acuerdo firmado a finales de 1947, por el cual reembolsaba 56 francos por cada consulta médica. El libro de oro del hospital, que recoge sesenta dedicatorias de los visitantes entre el 20 de mayo de 1948 y el 10 de septiembre de 1950, nos ofrecen una fuente fundamental de información para conocer los apoyos internacionales. Entre los demás benefactores podemos destacar al norteamericano Edward Barsky, médico de la Brigada Abraham Lincoln, que presidió la Joint Anti-Fascist Refugee Committee (JAFRC), que aportaba 370.000 francos mensuales para el mantenimiento del hospital entre 1945 y 1947, distribuidos a través de la organización humanitaria Unitarian Service Committee (USC). Recibió el apoyo de profesores de la facultad de medicina de Toulouse como Stephane Barsony, Garipuy, Tauber y Champagnac, destacando el apoyo del cirujano y miembro del PCF Joseph Ducuing. Todas las entidades colaboradoras serán recogidas en la sección "Nuestros Amigos" de la revista del hospital, destacando la Casa de Galicia de Unidad Gallega de Nueva York, la Spanish Refugee Appeal de Vancouver, la Comisión de Asistencia de Cuba, La Comisión de Ayuda al hospital Varsovia de México y otros, además de ayuntamientos de ciudades importantes como Glasgow, Varsovia o la propia Toulouse. El alcalde de la ciudad de Zurich y la Centrale Sanitaire Suisse contribuyeron a la dotación del centro donando un aparato de rayos $\mathrm{X}$

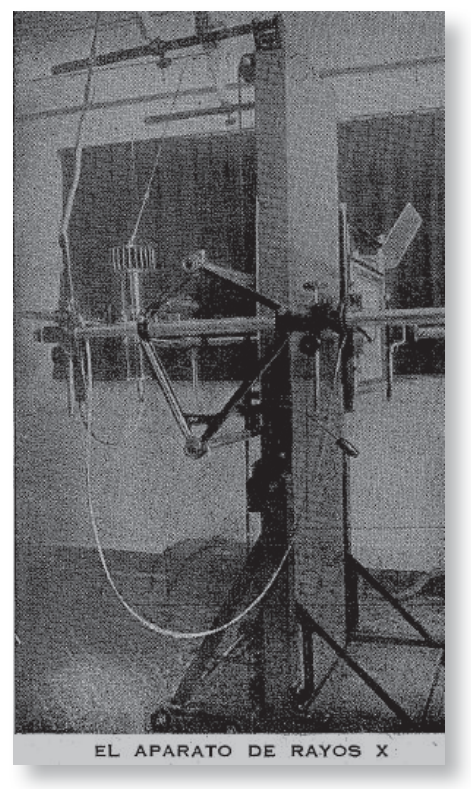

(Foto 3: Equipo de Rayos X donados por la Centrale Sanitaire Suisse. Fuente: Anales del Hospital Varsovia n5). 
Ante los rumores de tratarse de un refugio de comunistas la USC enviará a Persis Miller como delegada a Toulouse, que hará una reorganización del personal del hospital y licenciará al Dr. Torrubia como director, imponiendo al Dr. Parra. Pike (1984) afirma que Persis Miller fue informada de que "ciertos miembros del personal, especialmente el Dr. Víctor Viladrich y el enfermero Félix Bermejo Rodríguez eran toxicómanos y el P.C.E. había tomado el control total del hospital" (Pike, 1984: 175).

Como consecuencia de las tensiones internas y del inicio de la guerra fría a inicios de 1948 todo el comité de dirección de la JAFRC fue detenido en EEUU bajo la acusación de actividades antiamericanas y en febrero de 1948 la USC retira su apoyo al proyecto. Las JAFRC tendrán que crear la Spanish Refugee Appeall para poder continuar su aportación económica (Villar-Basanta, 1997: 77).

Durante la gestión de Vicente Parra, la dirección del hospital ahorró 3,5 millones de francos, reservados para acometer la ampliación del hospital, según Pike para una maternidad. La Amicale le exige la entrega de la suma ahorrada y ante la insumisión será cesado en febrero de 1948, siendo sustituido por Dr. Bosch.

A partir de marzo de 1948, bajo la dirección de Bosch el centro entrará en el período de dirección comunista. Según Pike se entregará la suma exigida por la Amicale, aunque Dolores Villar-Basanta, sin embargo discrepa en este aspecto ya que el centro acomete importantes obras a partir de julio de 1948, argumentando que éstas no habrían podido realizarse si hubiesen entregado los fondos ahorrados.

La situación de solidaridad por parte del gobierno francés, gracias al poder del PCF en el gobierno de la república para con el PCE comenzó a cambiar a partir de 1947, ante el de- but de la guerra fría. En mayo de 1947 los ministros comunistas del gobierno francés serían expulsados, perdiendo toda influencia a nivel gubernamental.

Según un documento de la prefectura de Toulouse con fecha del 4 de febrero de 1950 una purga del comité central del PCE había transcurrido el 8 de enero de ese mismo año expulsando del partido tanto al doctor Torrubia como a Pablo Cirera, ambos sanitarios del Hospital Varsovia de Toulouse.

\section{Un hospital moderno}

El hospital se constituye como un modelo de hospital republicano, con vistas a la implantación del mismo al retornar el régimen democrático a España. Por ello se potencia la formación del personal, tanto médico como auxiliar, la investigación, pese a las limitaciones económicas y técnicas y la publicación a través de la Revista Anales del Hospital Varsovia.

Se destina un presupuesto anual de 150.000 francos para la creación de una biblioteca de consulta. Se estimula también la realización de sesiones clínicas y cursos de actualización del personal sanitario, con el objetivo de "preparar mejor a nuestro personal para las tareas que tienen encomendadas hoy en Francia y mañana en España" (7). Estas sesiones clínicas se publicaron en la revista del hospital bajo el título "charlas clínicas del Hospital Varsovia". A ellas asistían no sólo los médicos y farmacéuticos, sino también las enfermeras y practicantes. Esta iniciativa formativa surge gracias a la experiencia de Josep Bonifaci en el Hospital Santa Creu de Barcelona. La instauración de la investigación como parte esencial de la formación profesional se inicia también en el período de dirección comunista, bajo una falta absoluta de medios. 
Entre las investigaciones hay que destacar los estudios realizados por Joaquín Vinyes sobre la avitaminosis C. Además el hospital fue pionero no sólo en el uso de la penicilina, proporcionada por el Unitarian Service Committee (USC), sino por los estudios de la acción terapéutica, efectos secundarios y de las dosis adecuadas de este medicamento.

Se estableció campañas de "Medicina social", un programa de acción sobre la salud de los refugiados españoles. Abordaron los problemas de desnutrición y campañas de lucha contra la tuberculosis y contra la sífilis, el cáncer y la mortalidad infantil. Para el programa de lucha contra el cáncer contaron con el apoyo del Centro Anticanceroso de Toulouse, dirigido por el cirujano Joseph Ducuing, miembro del PCF.

$\mathrm{Al}$ ser el centro sanitario de referencia para toda la comunidad exiliada, la demanda de atención se hará cada vez mayor, atendiendo pacientes de todo el territorio francés, incluso de las colonias. El aumento de las consultas externas obliga a crear un dispensario anexo en el Cours Dillón, instalado en unas barracas provisionales, que durante su primer año de funcionamiento realiza un total de 14.692 intervenciones. En un documento de la policía de Toulouse, con fecha del 7 de mayo de 1949 se registra la visita de Howard Fast al Hospital el 27 de abril de 1949, invitado por Francisco Bosch, director del hospital. Según sus declaraciones la persecución de la JAFRC en EEUU había impedido la donación de un millón de francos, necesarias para la construcción del nuevo dispensario (8). Finalmente, el nuevo dispensario se inaugurará el 30 de junio de 1949 , con un tamaño inicial de $90 \mathrm{~m} 2$ siendo ampliado ese mismo año para albergar un servicio de laboratorio de análisis biológicos con el nombre de Pabellón Thomas-Addis

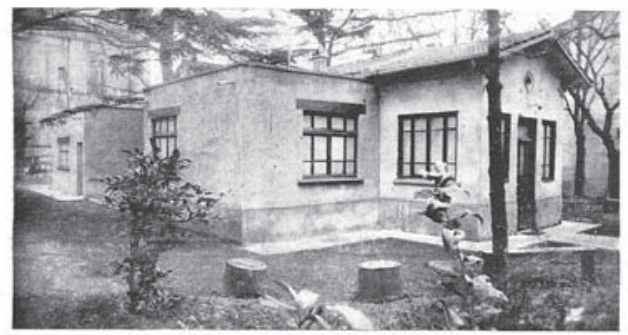

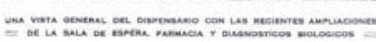

(Foto 4: Vista del nuevo dispensario. Fuente:

Revista Anales del Hospital Varsovia n7).

"En las dependencias del dispensario se prestan servicios de medicina general, tuberculosis, aparato digestivo, cirugía general, ginecología, garganta, nariz y oído, boca, ojos, piel, venéreo, infancia. Las dependencias de diagnóstico: rayos X, laboratorio clínico, metabolismo basal $y$ endoscopias, trabajan en el descubrimiento de las causas de las enfermedades y en comprobar el curso de estas últimas. Un aparato de diatermia, uno de rayos infra-rojos y otro de ultravioleta funcionan durante jornadas de 9 horas para devolver la salud a los enfermos. La sección de pequeñas curas aplica las inyecciones, hace las vacunaciones, los sondajes gástricos y duodenales, así como las curas de cirugía. Esta misma sección es la encargada de la toma de sangre a todo enfermo nuevo, dentro del marco de la búsqueda sistemática de la sífilis"(9)

María Gómez Álvarez, cirujana hija del odontólogo Ismael Gómez Carneado trabajó en el hospital hasta 1949. En el número 3 de la revista Anales del Hospital, de 1949, deja testimonio de la limitación de medios y de las mejoras realizadas en el centro hospitalario:

"Como no podía ser por menos, se pensó en instalar un servicio de cirugía, la falta de instrumental y de capacidad del edificio hacían de esta necesidad, casi una ilusión, pero poco a poco se instaló un quirófano, que hoy ha sido mejorado, de forma que no tiene nada que envidiar a 
hospitales modernos y se acopló a él un equipo quirúrgico formado de compatriotas e integrado por una enfermera, destinada a la esterilización e instrumental, otra encargada de la limpieza, cuidado de salas y asistencia mecánica de los enfermos; un practicante, que al mismo tiempo que hace el servicio de otras salas de medicina, se encarga de la anestesia general, un médico ayudante y yo como cirujano, que además de las intervenciones hago la consulta de cirugía en el consultorio. Como servicio complementario, tenemos el laboratorio, donde antes de ser operados los enfermos, se les realizan todos los análisis necesarios, Desgraciadamente la poca capacidad del Hospital hace que el servicio de cirugía solo pueda contar con siete camas para mujeres y nueve para hombres."(10)

En julio de 1948, bajo la dirección del Dr. Bosch, aparecerá el primer número de la revista Anales del Hospital Varsovia.

\section{HOSPITAL VARSOVIA}

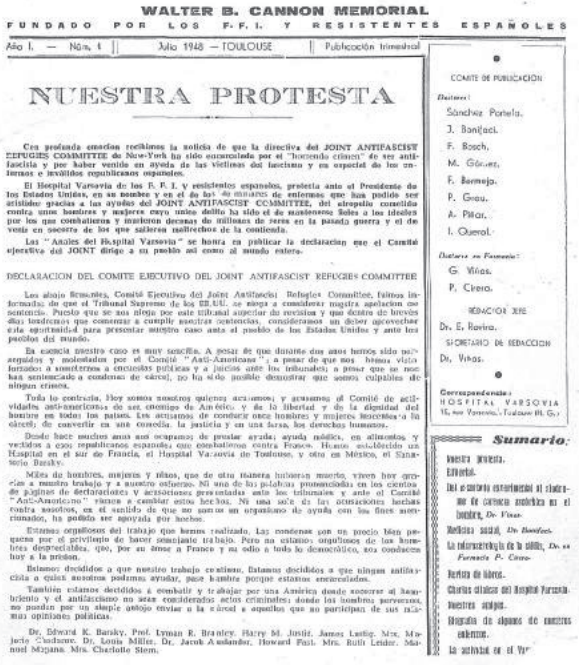

(Foto 5: Portada del primer número de la revista. Fuente: Revista Anales del Hospital Varsovia $\left.n^{\circ} 1\right)$.
Se trata de una publicación trimestral que tendría una vida de 9 números entre 1948 y 1950. Actualmente no existe ninguna colección que reúna todos los números pero un trabajo de recopilación dirigido por Alvar Martínez ha permitido reunirlos todos digitalizados y a disposición de los investigadores.

En el primer número, de julio de 1948 la editorial hace una declaración de objetivos del hospital "velar por la salud de toda la emigración española republicana en Francia”. Estimamos la influencia sobre esta revista del doctor Josep Bonifaci, quien había trabajado en España en el Hospital de la Santa Creu i Sant Pau de Barcelona, hospital que publicaba una revista de idéntico nombre a la del hospital Varsovia Anales del Hospital de la Santa Creu i Sant Pau (1927-1936), que recogía todas las actividades realizadas por el centro.

A partir del número de abril de 1950 se observa una reorganización del consejo editorial, reduciendo el número de consejeros a personas próximas a la dirección comunista del hospital.

\section{Un proyecto truncado: Operación Bolero- Paprika}

Fruto de la campaña anticomunista por el inicio de la guerra fría el centro hospitalario será acusado de ser "refugio de agentes soviéticos”. A finales del año 1945 el Comité de Actividades Antiamericanas de la Cámara de Representantes (HUAC) fija su atención en la Joint Anti-Fascist por la sospecha de tratarse de una organización afín al partido comunista. En 1946 las sospechas llegarán hasta los Unitarios, debiendo comparecer una delegación de la USC ante la HUAC en octubre de ese año para garantizar que no busca ningún fin político.

Las indagaciones de la policía acusarán a las instituciones financiadas por la USC de 
practicar discriminación a favor de los comunistas. Pelletier, prefecto del Alto Garona denuncia que los españoles se encuentran implicados en la mitad de los crímenes cometidos en Toulouse (11). En 1947 será el Comité de Actividades Antiamericanas quien impida la financiación gracias a los fondos aportados por las JAFRC.

Había un verdadero miedo a la amenaza que el apoyo de comunistas españoles podía suponer en el caso de que el PCF intentara hacerse con el poder en Francia en caso de un conflicto con la URSS. Esta situación se agravó tras la guerra de Corea y el descubrimiento el 17 de febrero de 1950 de un gran depósito de armas en Barbazan (Haute-Garonne). Alfred Coste-Floret, alcalde de Bagnères-de Luchon materializó con su declaración de marzo de 1950 el miedo al golpe comunista diciendo que las armas encontradas "no son destinadas a cazar a Franco, al contrario, según un plan establecido, a ayudar a los comunistas franceses en caso de guerra civil".

En el último número de la revista Anales del Hospital Varsovia, la editorial denuncia la persecución de los miembros de la JAFRC. Se detiene a Howard Fast, el cual declara en la revista del Hospital:

"El Comité de Actividades Antiamericanas nos ha exigido darles los nombres de los republicanos españoles que hemos ayudado, de suerte que al hacerlo nos hubiésemos convertidos en criminales asociados al abominable Franco. $Y$ porque nos hemos negado a ello vamos a la cárcel." (12)

La investigación policial francesa contó con la colaboración de los servicios de inteligencia franquistas “...según la información proveniente de la frontera española [del Servicio de Información y Policía Militar Franquista (SIPM)], esta asociación (refiriéndose a la USC) había sido sospechosa en 1947 de servir de cobertura a agentes de información norteamericanos a fin de facilitar sus actividades y sus desplazamientos"(13). Esto prueba la colaboración entre las autoridades franquistas y el gobierno francés, ante el interés de los franquistas de eliminar no solo toda amenaza de invasión por la frontera pirenaica, sino también de erradicar la labor social que se estaba realizando en el Hospital Varsovia impidiendo su financiación.

La visibilidad de los sanitarios de filiación comunista del hospital Varsovia le hizo objetivo de las pesquisas de los servicios policiales, de tal forma que los Archivos departamentales del Haute-Garonne conservan los informes policiales realizados entre 1948 y 1950 con motivo de desenmascarar a los agentes del Kominform trabajadores del hospital. Pelletier, en una carta al ministro del interior del 7 de febrero de 1950 acusaba al Dr.Bosch de ser agente del Kominform: "todo indica que el Dr. Bosch es uno de los agentes principales del brazo español del Kominform”, incluso va más allá y le propone "He pensado que es oportuno que la ley Billoux de agosto de 1945, no tenia fecha límite, pueda ser revocada; significará que los médicos españoles no tendrán más el derecho de ejercer, como los de Varsovia, lo que conllevará la clausura de este hospital. La población hospitalizada que no pasa de 50 enfermos podría ser, sin ninguna dificultad, admitidos en los diferentes hospitales de Toulouse..." (14)

El 7 de septiembre de 1950 son encarcelados en Toulouse 50 personas, entre ellas todo el equipo médico del hospital Varsovia.

La operación dirigida por el secretario de estado Eugène Thomas, con el nombre en clave de Bolero-Paprika supuso, según la información de la policía la detención y expulsión de Francia de 165 comunistas españoles (Alted y Domergue, 2003: 158). 


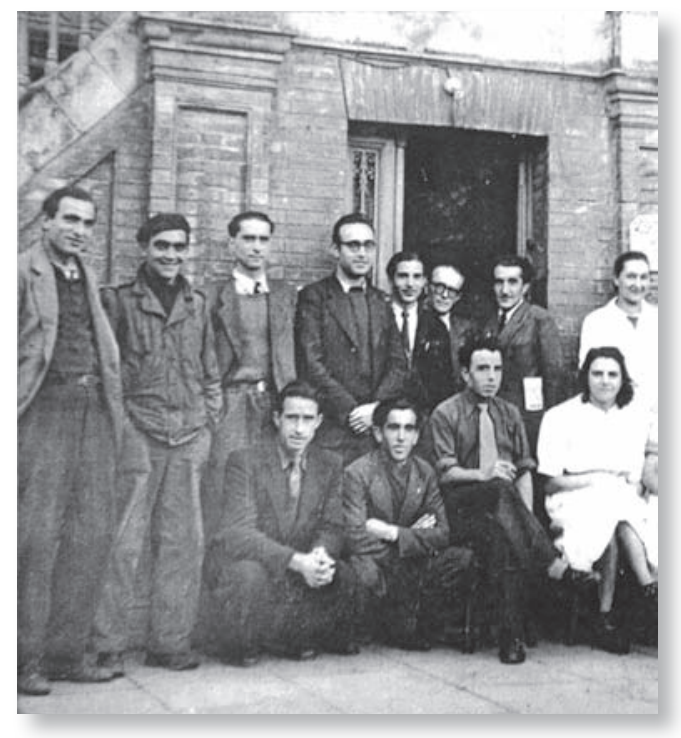

(foto 6: Personal español del hospital. Fuente:

Archivo del Hospital Joseph Ducuing).

"Todos los dirigentes conocidos del PCE en Francia, todo el personal del hospital Varsovia, todos los jefes de construcción y del servicio de la empresa Fernández-Valledor, así como los dirigentes de la Asociación de Antiguos miembros de las FFI y resistentes españoles y de otras asociaciones de obediencia comunista"

La operación tenía como objetivo desmantelar la quinta columna soviética en Francia, siendo justificada por el periódico France Soir en un artículo del 10 de septiembre de 1950 como:

"Los comunistas españoles del Sudoeste debian transformarse en guerrilleros... y bloquear a las tropas francesas el acceso a la región de los pirineos. Ellos debían controlar la región del atlántico a los pirineos esperando la llegada de los rusos" (15)

Sin embargo los miembros del PCE habían sido prevenidos de la operación por la prensa (16) y ningún documento interesante fue encontrado en la operación. El PCE, el PSUC y otras organizaciones, entre ellas la Amicale fueron declaradas ilegales, esta última disuelta el 7 de octubre de 1950. La mayor parte de los detenidos fueron expulsados a países de Europa del este a Córcega o a África del Norte.

\section{Recogida del testigo: profesor Ducuing.}

Consecuencia directa de la operación Bolero Paprika fue el cese de la actividad asistencial del Hospital Varsovia. Sin embargo un grupo de médicos franceses, liderados por el profesor Joseph Ducuing recogerán el testigo de la Amicale. Médicos como Champagnac, Lapeyrère y Tauber aparecen a partir de noviembre de 1950 como responsables de los pacientes ingresados. El ministerio del Interior ordena en una misiva firmada el 7 de octubre de 1950 la disolución de la Amicale y la liquidación de los bienes en el plazo de un mes. Se constituye la Societé Nouvelle Hôspital Varsovie, que finalmente adquirió las instalaciones el 6 de enero de 1951 por 300.000 francos (Garipuy, 1987: 32).

El propietario del castillo, Henri Maigne puso en venta el castillo, por la suma de seis millones de francos que la Nueva Sociedad pudo reunir gracias al apoyo financiero del Partido Comunista Francés, firmando la venta el 12 de febrero de 1952. El apoyo del PCF aseguró la pervivencia del centro, con continuidad asistencial hasta nuestros días.

\section{CONCLUSIONES}

Independientemente de las tensiones por el control del centro hospitalario el Hospital Varsovia fue un foco de investigación, heredero de la tradición de la II Republica y un refugio para miles de españoles exiliados, un centro que atendía a enfermos españoles, dirigido y atendido por sanitarios españoles. El proyecto se vio truncado por el viraje de la política internacional pero consiguió salvaguardarse 
gracias a la implicación de médicos franceses y al apoyo económico del PCF, asegurando su supervivencia hasta nuestros días. La reactivación de las investigaciones sobre el centro hospitalario y la apertura de nuevas líneas de trabajo preconizan un futuro halagüeño en el estudio del exilio sanitario español.

\section{NOTAS}

1- Guerra, F. (2003) La medicina en el exilio republicano. Madrid, Universidad de Alcalá. pp.204-206

2- Rodríguez, E. (2005) Els maquis. Cossetánia Edicions, Valls p.71. (traducción propia).

3- (1950) Essai biographique de l'hôpital Varsovie. pp.1112 (traducción propia).

4- (1950) Essai biographique de l'hôpital Varsovie. p.6 (traducción propia).

5- (1949) Editorial. Anales del Hospital Varsovia 4, 1

6- Widmaier, O. (2003). Picasso: retratos de familia. Algaba, Madrid p.136.

7- (1948) Revista Anales del Hospital Varsovia 1, 8.

8- Archivo Departamental Haut-Garonne. 2042 w292

Dossier 1 Chemise 3

9- (1949) Editorial. Anales del Hospital Varsovia 6, 1.

10- Gómez, M. (1949). Servicio de cirugía. Anales del Hospital Varsovia 3, 6.

11- Archivo Departamental Haute-Garonne. No1725 (10 febrero 1948).

12- (1950) Editorial. Anales del Hospital Varsovia 9, 1.

13- Archivo de la Prefectura de Policía, Paris. GA U3. Univarian Service Committee. (traducción propia).

14- CAC, Fontainebleau, MI, 19890576, Información del prefecto Pelletier (7 febrero 1950) (traducción propia).

15- En prensa: France Soir. 10 de septiembre de 1950 (traducción propia).

16- En prensa: La Dépeche du Midi, 6 septembre 1950 (traducción propia)

\section{BIBLIOGRAFÍA}

- Alted, A.; Domergue, L. (2003) El exilio republicano español en Toulouse, 1939-1999. Universidad Nacional de
Educación a Distancia, Madrid

- Alted, A. (1991) La Cruz Roja Republicana Española en Francia, 1945-1986. Historia Contemporánea 6, 223249

- Cubero, J. (2003) Les Républicains espagnols. Cairn, Toulouse:. 316-319

- Dreyfus-Armand,G. (1999) L'Exil des républicains espagnols en France de la guerre civile à la mort de Franco. Albin Michel, Paris.

- Garipuy, J. (1987) L'Hôpital Joseph Ducuing et son projet: Histoire, realite et image d'aujourd 'hui. Project These Doctorat. Université Paul Sabatier, Toulouse.

- Guerra, F. (2003) La medicina en el exilio republicano. Universidad de Alcalá, Madrid:. 215-302.

- Lillo, N. (2007) El PCE en Francia: relaciones con el PCF y evolución. 1945-1975. En Bueno, M.; Hinojosa, J.; García, C. (coord.). Historia del PCE, I. Fundación de Investigaciones Marxistas, Madrid:. 83-99.

- Martínez, À. (2010) Exili, medicina i filantropía. L'Hospital Varsòvia de Tolosa de Llenguadoc (19441950). Editorial Afers, Barcelona.

- Martínez, À. (2011) L'Hôpital Varsovie. Exil, médecine et résistance (1944-1950). Loubatières, Portet-sur-Garonne.

- Molanes, P. (2011) El Hospital Varsovia. Exilio, medicina y resistencia (1944-1950) [Comentario de Texto]. Temperamentvm 14. Available http:www.index-f.com/ temperamentum/tn14/t1011.php. downloaded 15th january 2011.

- Moran,G. (1986) Miseria y grandeza del Partido Comunista de España. 1939-1985. Planeta, Barcelona:. 92-95

- Pike, D. (1984) Jours de gloire, jours de honte: le parti communiste d'Espagne en France depuis son arrivée en 1939 jusqu'à son départ en 1950. Société d'éditions d'enseignement supérieur, París

- Pike, D. (1969) Vae victis!: Los republicanos españoles refugiados en Francia, 1939-1944. Ruedo Ibérico, París.

- Villar-Basanta, D. (1997) L'Hôpital Varsovie, 1944-1950. Memoire de D.E.A. Université Toulouse-Le Mirail, Toulouse. 